\title{
Three-dimensional photonic confinement in imprinted liquid crystalline pillar microcavities
}

Marco Dusel ${ }^{1}$, Simon Betzold ${ }^{1}$, Sebastian Brodbeck ${ }^{1}$, Stefanie Herbst ${ }^{2}$, Frank Würthner ${ }^{2}$, Daniel Friedrich ${ }^{3}$, Bert Hecht $^{3}$, Sven Höfling ${ }^{1,4}$, and Christof P. Dietrich ${ }^{1 \mathrm{a})}$

${ }^{1}$ Technische Physik, University of Würzburg, Am Hubland, 97074 Würzburg, Germany

${ }^{2}$ Institut für Organische Chemie and Center for Nanosystems Chemistry, University of Würzburg, Am Hubland, 97074 Würzburg, Germany

${ }^{3}$ Nano-Optics and Biophotonics Group, University of Würzburg, Am Hubland, 97074 Würzburg, Germany and

${ }^{4}$ SUPA, School of Physics and Astronomy, University of St Andrews, North Haugh, KY16 9SS St Andrews, United Kingdom

(Dated: 3 May 2017)

We demonstrate the feasibility of a thermal imprint technology capable of structuring organic thin films with liquid crystalline properties forming feature sizes on a several micrometer scale. The imprint technique can directly be applied onto a variety of substrates including dielectric mirrors. The so fabricated three-dimensional microcavities have lateral extensions up to $20 \mu \mathrm{m}$ and heights between 1 and $5 \mu \mathrm{m}$. Exemplarily, pillar microcavities were produced wherein three-dimensional photonic confinement is observed by the formation of $0 \mathrm{D}$ cavity mode patterns. The imprint technique further favors the formation of hemispherical pillar geometries rather than cylindrical pillars resulting in equidistant mode spacings of transversal cavity modes.

Although organic dyes have already been used as gain medium in laser cavities for more than fifty years, microcavities with active organic medium are still in the spotlight of modern research activities as they hold great potential for emergent applications such as lab-on-a-chip architectures or sensing devices ${ }^{1}$. This is first and foremost related to the versatility and flexibility that is offered by organic compounds. They not only provide spectral tunability and chemical customizability, they can also be fabricated at low costs and with simple processing techniques. The simplest organic cavity design is the planar microcavity that is composed of the active organic layer sandwiched between two highly reflective mirrors (either metallic or dielectric). In this planar geometry, organic photon lasing ${ }^{2,3}$ as well as polariton condensation ${ }^{4-6}$ was already demonstrated. More elaborated cavity concepts include distributed feedback (DFB) gratings ${ }^{7}$, whispering gallery resonators ${ }^{8}$, photonic crystals ${ }^{9}$ or hemispherical cavities ${ }^{10}$. While those provide multi-dimensional photonic confinement, planar cavity designs achieve confinement only in the out-of-plane direction. In order to decrease the mode volume for planar cavities anyhow, it is necessary to provide a photonic modulation in plane by either structuring the excitonic or dielectric part of the organic microcavity. So far, this has been achieved by incorporating additional metal layers ${ }^{11}$ into the cavity or by laser writing ${ }^{12}$.

In this letter, we present a method to create threedimensional photonic confinement in an organic microcavity system: thermal imprint technology using a liquid crystalline dye. Imprinted structures were so far limited to a few hundreds of nanometers in feature size. Here,

a)Electronic mail: christof.dietrich@physik.uni-wuerzburg.de we make use of the liquid-crystalline nature of the recently developed organic compound MEH-PBI (bearing twelve 2-ethylhexyl substituents at the perylene bisimide core $)^{13}$. In the liquid crystalline phase, a pre-patterned stamp is pressed into a layer of the organic material creating micropillars with micrometer-sized dimensions. Imprinting directly on dielectric mirrors and capping the micropillars by a thin gold layer creates three-dimensional photonic confinement. The fabricated pillar microcavities show pronounced 0D cavity mode patterns with equidistant transversal modes indicating rather hemispherical than cylindrical symmetry. This assumption is confirmed by a combination of atomic force microscopic (AFM) measurements as well as emission spectrum analysis.

Figure 1(b-g) illustrates the fabrication process of both the stamp used for imprinting micropillars and the microcavity. The patterning of the stamp requires a GaAs substrate coated with a photoresist (ARU 4030) being illuminated with UV light through a chrome mask. The irradiated parts are subsequently developed, coated with a metal protection layer $(\mathrm{Ti} / \mathrm{Cr})$ and dry-etched leaving holes with predefined diameters and depths. The stamp is then heated to $125^{\circ} \mathrm{C}$ and pressed (using a force of about $100 \mathrm{~N}$ ) into an equally heated layer of MEHPBI spin-coated on top of a dielectric mirror (10 pairs of $\mathrm{SiO}_{2} / \mathrm{TiO}_{2}$, designed for a central wavelength of $620 \mathrm{~nm}$ ). Note that the presented imprint technique can be conducted using other stamp materials as long as the sidewall steepness of the mask holes is sufficiently high.

MEH-PBI combines two exceptional properties of organic semiconductors: the simultaneous formation of $\mathrm{J}$ aggregates and the existence of a liquid crystalline phase. This was achieved by tetra-substituting the bay position of the PBI core with four dendritic wedges with branched 2-ethylhexyl groups ${ }^{13}$. The molecular structure of MEH- 
(a)

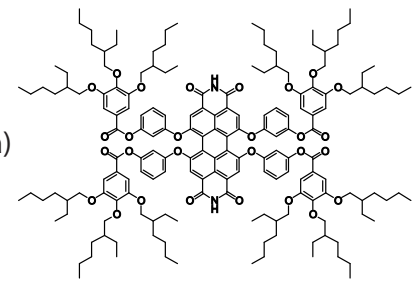

(b)

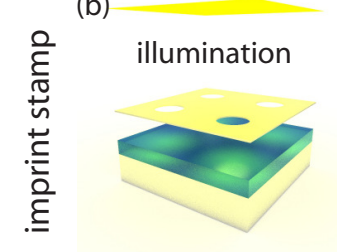

(c)

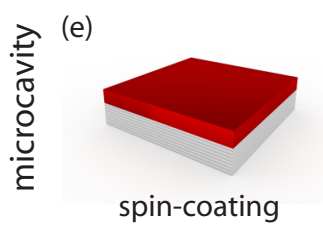
development

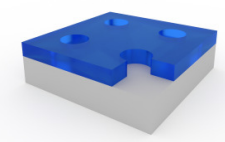

(f)

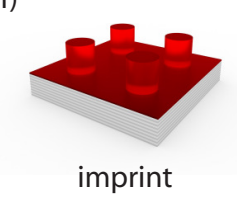

(d)

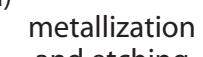
and etching

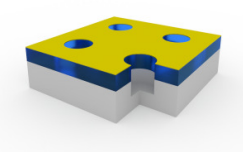

(g)

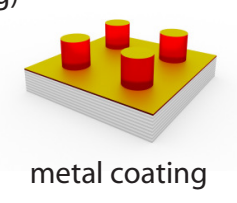

FIG. 1. Molecular structure of MEH-PBI (a) and fabrication steps for imprint stamp (b-d) and pillar microcavities (e-g). The imprint stamps are composed of an GaAs substrat (gray) and a homogeneous layer of negative photoresist (blue). b) The samples are illuminated by UV light through a patterned chrome mask, (c) the photoresist is subsequently developed and the stamp is metalized. (d) Deep holes are achieved by means of dry etching. Pillars are produced by (f) imprinting the fabricated stamp into (e) a liquid crytalline thin film (red, spin-coated on a dielectric mirror). (g) Pillars are finally capped by a gold coating forming the metal-DBR cavity.

PBI is shown in Fig.1(a). As result of their liquid crystallinity, MEH-PBI molecules are to a certain extend viscous and subsequently fill the pre-defined holes of the stamp. After cooling down and removal of the stamp, organic micropillars with diameters ranging from 7 to $20 \mu \mathrm{m}$ (depending on the stamp pattern) and heights between 1 and $5 \mu \mathrm{m}$ (depending on the organic layer thickness) remain. In principle, the pillar diameter is determined only by the mask hole diameter and can be much smaller than this (approximately $1 \mu \mathrm{m}$ ). Electron microscopic images of imprinted micropillar arrays can be seen in Fig.3(a-c) for different magnifications. Note that, besides a significant drop in emission efficiency from solution to solid state due to concentration-induced quenching, we do not observe any further noticeable decrease in efficiency due to the imprint process.

In a final step, the imprinted micropillars are capped by a 70-nm-thick gold layer to form a cavity between the bottom dielectric mirror (distributed Bragg reflector, DBR) and the top gold layer. The optical field distribution in such a structure is calculated for an organic layer height of $3.6 \mu \mathrm{m}$ and shown in Fig.2(a). Due to the increased cavity thickness as compared to conventional $\lambda / 2$-cavities, several nodes of the optical field lie within the organic layers. The resulting reflectivity spectrum is depicted in Fig.2(b) (black line) together with
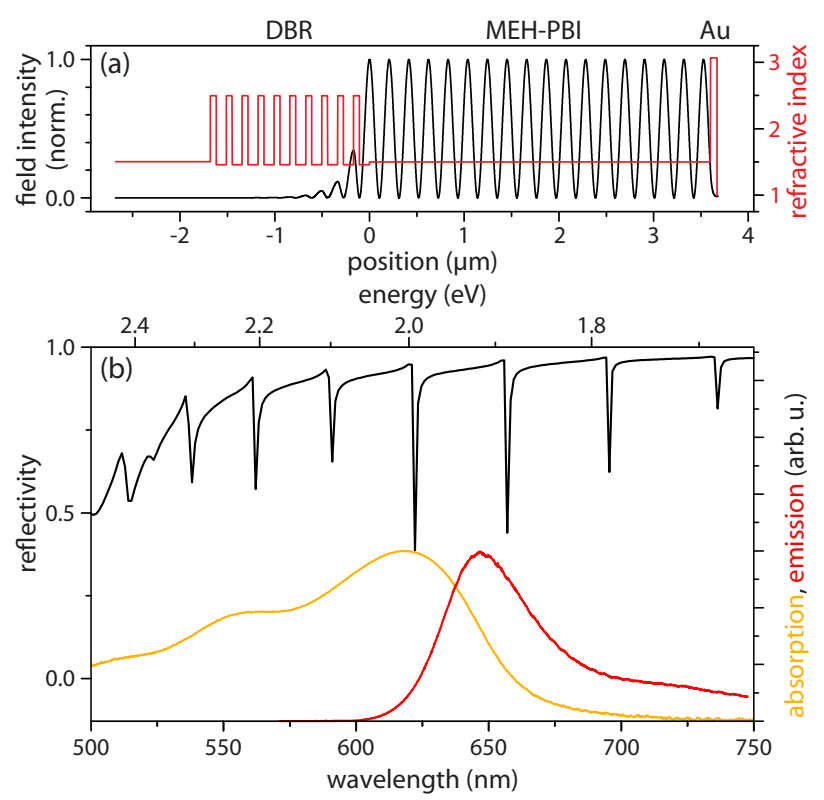

FIG. 2. (a) Optical field distribution (along the out-of-plane axis) inside the metal-DBR cavity filled with MEH-PBI (black line). The position-dependent refractive index used for calculating the field distribution is shown in red. (b) Calculated reflectivity of the metal-DBR cavity shown in (a) (black line) together with the normalized absorption (orange line) and emission (red line) spectrum of MEH-PBI in its solid state.

the main absorption (orange line) and emission (red line) bands of MEH-PBI in its solid state. Compared to measurements of MEH-PBI J-aggregates in solution ${ }^{13}$, the main absorption band of solid MEH-PBI is broadened which we attribute to increased disorder due to inhomogeneous arrangement of the phenoxyl substituents of densely packed PBI columns. However, the reflectivity of the metal-DBR microcavity remains high $(>90 \%)$ over the entire spectral range of main MEH-PBI absorption and emission resulting in optical cavity modes with theoretical quality factors as high as 1500 (determined from simulated reflectivity in Fig.2(b)).

The fabricated micropillars were subsequently excited by a $532 \mathrm{~nm}$ diode laser $(50 \mu \mathrm{W})$ through a microscope objective $(50 \mathrm{x}, N A=0.42)$. The angular-resolved emission of the pillar microcavities was spectrally dispersed in a spectrometer with $0.06 \mathrm{~nm}$ spectral resolution and monitored in Fourier imaging configuration on a 2D detector chip. The emission pattern of a pillar microcavity with a diameter of $d=8.2 \mu \mathrm{m}$ and a height of $3.6 \mu \mathrm{m}$ can be seen in Fig.3(d). MEH-PBI exhibits pronounced self-absorption within a cavity due to overlapping emission and absorption spectrum. This leads to a shift of maximum microcavity emission to longer wavelengths. Fig.3(d) shows several families of quantized optical modes without energy dispersion (with experimental quality factors exceeding 500). The absence of a photonic dispersion confirms the presence of an effective three-dimensional optical confinement within the im- 
printed pillar microcavities. While the different families represent different longitudinal modes (with longitudinal mode numbers $l$ ), the different modes within a mode family are transversal modes with different transversal mode numbers $m$. Note that liquid crystals typically exhibit birefringent optical properties owing to the alignment of molecules. We observe the same effect in our structure (not shown), basically producing two sets of mode families with orthogonal polarization. Hence, the shown spectra are linearly polarized to select the ordinary modes.

We find that all transversal modes appear to maintain a constant mode distance between them. This contradicts the theoretical cylindrical pillar geometry that theoretically forms non-equidistant transversal mode patterns according to the roots of the spherical Bessel functions. This suggests that we have a broken symmetry along the pillar axis imposed by the imprint process. This can be explained by the liquid-crystalline phase of MEH-PBI. Since the organic compound is viscous, it hypothetically forms a curved surface profile inside the mask holes due to capillary forces and therefore creates a hemispherical micropillar. The energies of transversal and longitudinal modes in hemispherical microcavities then linearly scale with mode numbers $m$, the energetic mode separation $\Delta E_{m}$ between neighboring transversal modes is therefore constant in agreement with our observation.

In general, mode energies and their spectral shifts $\Delta E_{m}$ are related to the mode number change $\Delta m^{14}$ according to

$$
\Delta E_{m}=\frac{\hbar c \Delta m}{n_{\mathrm{eff}} L_{\mathrm{eff}}} \arccos \left[\sqrt{1-\frac{d_{\mathrm{cav}}}{R_{\mathrm{C}}}}\right]
$$

with $n_{\text {eff }}$ being the effective cavity index of refraction, $L_{\text {eff }}$ being the effective cavity length and $R_{\mathrm{C}}$ being the radius of curvature of the hemispherical mirror. By taking into account Eq.1 and Laguerre polynoms (that represent solutions of the wave equation for circular in-plane symmetry), we are able to recalculate the observed mode pattern. With $n_{\mathrm{eff}}=1.5, L_{\mathrm{eff}}=d_{\mathrm{Cav}}+L_{\mathrm{DBR}}\left(d_{\mathrm{Cav}}=\right.$ cavity thickness; $L_{\mathrm{DBR}}=$ penetration depth into dielectric mirror) and $R_{\mathrm{C}}=25 \mu \mathrm{m}$, we can nicely reproduce the emission pattern from Fig.3(d) in Fig.3(e).

To validate the hemispherical assumption, we have performed AFM measurements of individual micropillars. A representative height profile of a micropillar with $18.5 \mu \mathrm{m}$ diameter is shown in Fig.4(a) together with a full three-dimensional representation of the micropillar derived from the scan. The profile shows non-vertical side facets of the micropillars which we associate to a measurement artifact (probably related to the finite speed of the AFM tip during scanning and its lateral extension). However, the surface appears to be curved. By fitting the top surface profile using a spherical geometrical formula, we deduce the actual radius of curvature $R_{\mathrm{C}}$ of the investigated micropillars. Those determined values are plotted versus pillar diameter in Fig.4(b) (red circles) together with data points derived from several emission spectra using Eq.1 (black diamonds). It is obvious from



FIG. 3. (a-c) Electron microscopic images of imprinted micropillars. The scale bars represent $150 \mu \mathrm{m}, 50 \mu \mathrm{m}$ and $12 \mu \mathrm{m}$, respectively (from left to right). (d) Microcavity emission of a micropillar with $d=8.2 \mu \mathrm{m}$ and $h=3.6 \mu \mathrm{m}$. Numbers in white indicate mode numbers $l$ and $m$ of longitudinal and transversal modes, respectively. (e) Calculated emission spectrum of a hemispherical microcavity using $n_{\mathrm{eff}}=1.5, L_{\mathrm{eff}}=$ $4.6 \mu \mathrm{m}$ and $R_{\mathrm{C}}=25 \mu \mathrm{m}$.
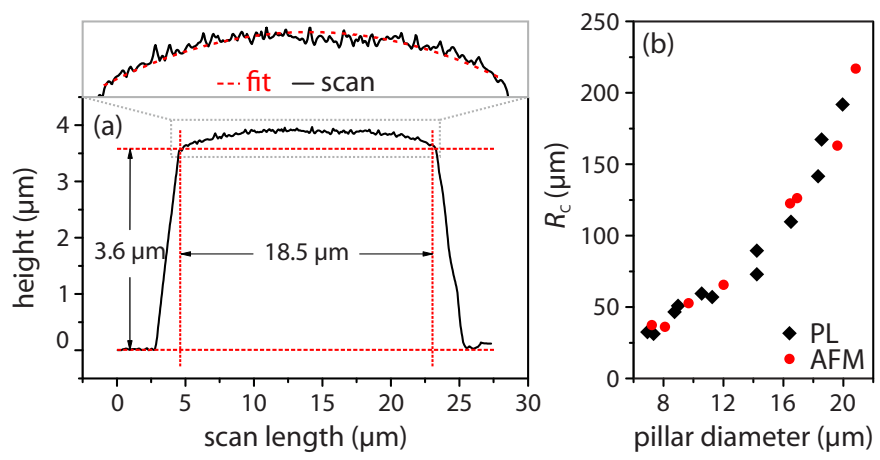

FIG. 4. (a) Atomic force microscopic (AFM) height profile of an imprinted liquid-crystalline micropillar. The inset shows the spherical fit (red dashed line) to the curved pillar surface (black line). (b) Radius of curvature vs. pillar diameter of imprinted micropillars. The radii were calculated from emission spectra (black diamonds) or measured by atomic force microscopy (red circles), respectively

Fig.4(b) that both sets of data points follow the same dependency confirming the validity of our assumptions. Note that fabrication errors during the imprint process can also alter the mode pattern. They might induce a degree of ellipticity leading to a lifting of transversal mode degeneracy (absent in our case). In general, fabrication errors induce Q factor quenching.

In conclusion, we have demonstrated the feasibility of thermal imprint technology to fabricate organic pillar microcavities providing three-dimensional optical confinement. Pillars were imprinted using a stamp into an active 
layer of liquid-crystalline MEH-PBI. The imprinted pillars have diameters up to $20 \mu \mathrm{m}$ and heights up to $5 \mu \mathrm{m}$. By imprinting the pillars directly on top of dielectric mirrors and capping them by a thin gold layer, we achieve microcavities with photonic confinement in all three spatial directions. Whereas confinement along the pillar axis is realized by reflections between the mirrors, in-plane confinement is obtained by total internal reflection at the steep side facets of the pillars. The emission of imprinted micropillars shows pronounced optical resonances of $0 \mathrm{D}$ cavity modes. Their mode separation is constant contradicting cylindrical symmetry. We assumed a rather hemispherical pillar geometry being confirmed by a combination of AFM measurements and spectrum analysis in accordance with optical resonator theory.

The achieved feature sizes of the demonstrated imprint technique exceed commonly accomplished imprint values by an order of magnitude being promising for scalable and parallel structuring of larger areas. It may therefore serve as a building block for more complex microcavity designs including pillar chains and lattices as well as waveguides and networks towards quantum simulation and computing experiments. The mode patterns with equidistant mode separation energies in the range of a few meV might further render the use of imprinted micropillars in the field of $\mathrm{THz}$ emitters interesting. With respect to boosting mode quality factors, the imprint technology can also easily be combined with the open microcavity approach $^{15}$ enabling cavities with a top and bottom DBR mirror.

We acknowledge fabrication of masks and assistance in micropillar imprint by A. Wolf. Sv.H. also acknowledges financial support by the EPSRC "Hybrid Polaritonics" Grant (EP/M025330/1). F.W. further thanks the Deutsche Forschungsgemeinschaft (DFG) for financial support (WU317/18-1).

\section{REFERENCES}

${ }^{1}$ A.J.C. Kuehne and M.C. Gather, Chem. Rev. 116, 12823 (2016).

${ }^{2}$ N. Tessler, G.J. Denton, and R.H. Friend, Nature 382, 695 (1996).

${ }^{3}$ V. Bulović, V.G. Kozlov, V.B. Khalfin, S.R. Forrest, Science 279, 553 (1998).

${ }^{4}$ K.S. Daskalakis, S.A. Maier, R. Murray, and S. Kéna-Cohen, Nat. Mater. 13, 271 (2014).

${ }^{5}$ J.D. Plumhof, T. Stöferle, L. Mai, U. Scherf, and R.F. Mahrt, Nat. Mater. 13, 247 (2014).

${ }^{6}$ C.P. Dietrich, A. Steude, L. Tropf, M. Schubert, N.M. Kronenberg, K. Ostermann, S. Höfling, and M.C. Gather, Sci. Adv. 2, e1600666 (2016).

${ }^{7}$ M.D. McGehee, M.A. Diaz-Garcia, F. Hide, R. Gupta, E.K. Miller, D. Moses, and A.J. Heeger, Appl. Phys. Lett. 72, 1536 (1998).

${ }^{8}$ T. Grossmann, S. Schleede, M. Hauser, M. Brøkner Christiansen, C. Vannahme, C. Eschenbaum, S. Klinkhammer, T. Beck, J. Fuchs, G.U. Nienhaus, U. Lemmer, A. Kristensen, T. Mappes, and H. Kalt, Appl. Phys. Lett. 97, 063304 (2010).

${ }^{9}$ M. Meier, A. Mekis, A. Dodabalapur, A. Timko, R.E. Slusher, J.D. Joannopoulos, and O. Nalamasu, Appl. Phys. Lett. 74, 7 (1999).

${ }^{10}$ D. Urbonas, T. Stöferle, F. Scafirimuto, U. Scherf, and R.F. Mahrt, ACS Photonics 3, 1542 (2016).

${ }^{11}$ R. Brückner, A.A. Zakhidov, R. Scholz, M. Sudzius, S.I. Hintschich, H. Froeb, V.G. Lyssenko, and K. Leo, Nat. Photonics $6,322(2012)$.

${ }^{12}$ C.P. Dietrich, M. Karl, J. Ohmer, U. Fischer, M.C. Gather, and S. Höfling, Adv. Mater. 1605236 (2017), DOI: 10.1002/adma.201605236.

${ }^{13}$ S. Herbst, B. Soberats, P. Leowanawat, M. Lehmann, and F. Würthner, Angew. Chem. 56, 2162 (2017).

${ }^{14}$ A.E. Siegman, Lasers, University Science Books (Mill Valley, 1986).

${ }^{15}$ M. Trupke, E.A. Hinds, S. Eriksson, E.A. Curtis, Z. Moktadir, E. Kukharenka, and M. Kraft, Appl. Phys. Lett. 87, 211106 (2005). 